\title{
EFFICIENCY OF METHYLENE BLUE MEDIATED PHOTODYNAMIC THERAPY verSUS INTENSE PULSED LIGHT IN THE TREATMENT OF ONYCHOMYCOSIS IN THE TOENAILS
}

\begin{tabular}{|r|l|}
\hline Journal: & Photodermatology, Photoimmunology \& Photomedicine \\
\hline Manuscript ID & PHOTO-OA-04-18-1014.R1 \\
\hline Manuscript Type: & Original Article \\
\hline Date Submitted by the Author: & n/a \\
\hline Complete List of Authors: & $\begin{array}{l}\text { Alberdi, Enrique } \\
\text { Gómez, Clara; Spanish National Research Council, CSIC, }\end{array}$ \\
\hline Keywords: & $\begin{array}{l}\text { Onychomycosis, Photodynamic Therapy, Methylene blue, Intense pulsed } \\
\text { light }\end{array}$ \\
\hline \multicolumn{2}{|l}{} \\
\hline
\end{tabular}


Title: EFFICIENCY OF METHYLENE BLUE MEDIATED PHOTODYNAMIC THERAPY versus INTENSE PULSED LIGHT IN THE TREATMENT OF ONYCHOMYCOSIS IN THE TOENAILS.

\author{
Running healing: Photodynamic therapy vs Intense pulsed light for onychomycosis treatment \\ Authors: Enrique Alberdi ${ }^{1}$, Clara Gómez ${ }^{2 *}$ \\ 1 Consulta privada Enrique Alberdi, Madrid, Spain \\ 2 Instituto de Química Física Rocasolano, Consejo Superior de Investigaciones Científicas, CSIC, \\ Madrid, Spain
}

\author{
Address of authors: \\ Clara Gómez (author for correspondence)*: \\ Instituto de Química Física Rocasolano \\ Consejo Superior de Investigaciones Científicas, CSIC \\ C/Serrano 119, 28006 Madrid \\ Phone number: +34 917459532 \\ Fax: + 915642431 \\ c.gomez@iqfr.csic.es
}

\title{
Financial disclosure/Acknowledgements
}

This research was supported by a grant from the Eugenio Rodríguez Pascual Foundation (Madrid, Spain) and by the Spanish Research Projects MINECO (Ref.: MAT 2017-83856-C3) and (MAT2015-68837REDT). Authors wish to thank Intra-Lock Iberia Spanish Enterprise for lending his Periowave ${ }^{\circledR}$ laser system and Dr. MJ Ortiz García for her input and suggestions while designing the study protocol. The author have no other relevant affiliation or financial involvement with any organization or entity with a financial interest in or financial conflict with the subject matter or materials discussed in the manuscript apart from those disclosed. 


\section{ABSTRACT}

Background: Photodynamic therapy (PDT) or Intense Pulsed Light (IPL) are efficient therapeutic methods in the treatment of superficial skin infections and thus they could be good options for Onychomycosis treatment, the most common nail disorder.

Methods: Forty patients, affected with different diagnosed types of Onychomycosis in nails of the first toe, were randomly divided into two groups of 20 patients to be treated by PDT or IPL. Nail plates were softened with urea $40 \%$ by occlusive dressing for $12 \mathrm{~h}$ during 3-7 days before treatments. Then 8 sessions separated by an interval of 2 weeks of a PDT protocol mediated by methylene blue (MB) and red laser diode (Periowave ${ }^{\circledR}, \lambda=670 \mathrm{~nm}, 200 \mathrm{~mW}$ ) or an IPL protocol based on 10 pulses $/ \mathrm{cm}^{2}$ (Dye-VL-F module, Alma Lasers, $\lambda=500-600 \mathrm{~nm}, 10 \mathrm{~J})$, were applied.

Results: Both treatments reduced significantly the Onychomycosis Severity Index (OSI) $(\mathrm{p}<0.05)$. In terms of complete cure: $70 \%$ (PDT) and $80 \%$ (IPL) of the patients reached it after 12 weeks posttreatment. No patient reported any adverse effects or complications, although in the IPL Group, some referred pain sensation during light irradiation and hematomas apparition.

Conclusions: PDT and IPL were effective for Onychomycosis cure of any etiology.

Keywords: Onychomycosis, Photodynamic therapy (PDT), Methylene blue (MB), Intense pulsed light (IPL)

\section{Summary statement:}

Innovative therapies as alternatives for Onychomycosis treatment are needed in order to avoid the collateral hepatic side effects of the conventional one. The present study shows that PDT/MB and IPL are effective, well tolerated and not associated to adverse effects. After 3 months of PDT/MB or IPL treatment $70 \%$ and $80 \%$ of complete cure is reached, respectively. 


\section{INTRODUCTION}

Onychomycosis is a fungal nail infection, caused by dermatophytes (responsible for $90 \%$ ). Trichophyton rubrum or Trichophyton mentagrophytes (identified as the most frequent causative pathogens), yeasts (responsible in 5\% of cases) and non-dermatophyte fungi (responsible in 3\% of cases), affecting the nail bed, matrix, and/or plate, mainly in toenails in adults $(1,2)$. It is very difficult to diagnose and treat $(2,3)$. This condition accounts for one-third of all skin infections and is increasing in prevalence. A recent survey indicates that 2 to $18 \%$ of the world's population is affected, accounting for $40 \%$ of all nail disturbances (3). Onychomycosis can lead to serious infections such as osteomyelitis and cellulitis, particularly in diabetics (4). More importantly, onychomycosis can represent a serious health problem in immunocompromised patients such as those infected by HIV.

Once Onychomycosis is diagnosed, the available treatment modalities that may be considered are topical therapy, systemic (oral) therapy and surgical intervention $(5,6)$. Topically applied drugs are generally unable to penetrate the entire nail unit (7). Systemic drug administration, such as Griseofulvin, Ketoconazole, Terbinafine, Itraconazol may require up to 12 months of treatment. In addition, these agents may be associated with risk of toxicity and possible serious drug interactions. There is also concern about the development of resistance, especially when these agents are used in the long term (8). Surgical intervention is not a major benefit, since it can generate pain, disfigurement and permanent healing potential (7). Onychomycosis is a therapeutic challenge because the nails are non-vascularized structures that compromise the penetration of the administered drugs, while favoring a very slow growth (9). All this, associated with the high incidence of this type of infection, shows the importance of developing new technologies and treatment options.

In the last years, laser technology has positioned to be an alternative option for Onychomycosis treatment. Lasers through selective photothermolysis exert fungicidal effects by photothermally heating fungal mycelium (10). Chitin, xanthomegnin and melanin as fungal chromophore targets allow exert the laser fungicidal effects. $532 \mathrm{~nm}$ - Q-switched Nd:YAG laser on fungal colonies showed inhibitory effect due to its selective absorption by the red pigment of xanthomegnin (11). The presence of xanthomegnin may explain the sensitivity of T. rubrum to 532-nm range. Q-switched Nd:YAG laser at 1,064nm showed also inhibitory effect on the colonies treated. It is known that Trichophyton species contain melanin in their cell wall and it selectively absorbs this wavelength (12). 
The photodynamic method is a promising technique for both diagnosis and therapy of numerous diseases. Photodynamic therapy (PDT) requires the presence of 3 basic elements: 1) Photosensitizer (PS); 2) light source causing photoexcitation of PS; 3) presence of dissolved oxygen in the treated tissue. PDT is based on the interaction between these components and their effectiveness depends on their cooperation (13, 14). In PDT, the excited triplet state of the PS interacts with the diseased tissue molecules generating radicals that in the presence of the surrounding oxygen originate the reactive oxygen species (ROS). The excited triplet state of the PS can also directly interact with oxygen to form singlet oxygen $\left({ }^{1} \mathrm{O}_{2}\right)$, a highly reactive molecule that easily damages tissues. All these species generated are highly cytotoxic and cause damage on the mitochondria leading to a programmed death of the fungal cells $(15,16)$. PDT has certain characteristics that can be useful for the treatment of onychomycosis and therefore may be an advantageous therapy compared to the other therapies before described: it is not invasive, more convenient for patients, its target is only the diseased tissue and not the whole organism, it hardly has any side effects, can be combined with other treatments, its effect is faster than antifungal agents (action confined exclusively to the nail injury) and there is no data on possible resistance until now. The following is worth pointing out as well: the low cost of the instrumentation involved, the simplicity of the operation and the fast results obtained (17-19).

The intense pulsed light system (Intense Pulsed Light, IPL) is a high-power broadband light source compared to the monochromatic laser. IPL systems emit non-coherent polychromatic light (both spatially and temporally), between 400 and $1200 \mathrm{~nm}$, with a pulse duration of between 2 and $200 \mathrm{~ms}$. (20). The advantage of IPL systems is their great versatility, so with the same device it is possible to treat different conditions focusing on different chromophores (mainly hemoglobin and melanin) using different performance parameters such as wavelength, pulse duration, number of pulses, interval between pulses. In addition, the irradiated area per pulse is relatively large, which makes it possible to increase the speed of treatments (21). Initially, IPL systems were used for the treatment of vascular malformations such as port wine stains, angiomas, telangiectasias, etc. In the $90 \mathrm{~s}$, it began to have a place of greater weight in the photodermatological treatments, with the exception of the elimination of tattoos. In the dermatological field, IPL has numerous applications: benign pigmented lesions, vascular lesions, rosacea, acne vulgaris, photoepilation, photorejuvenation, hypertrophic scars, seborrheic keratosis, atopic dermatitis, cutaneous lupus erythematosus, hidradenitis suppurativa, recalcitrant viral warts, lichen planus, psoriasis in the body 
and in the nails (21). There are hardly any studies on its application in onychomycosis, only a small in vitro test using IPL at $420 \mathrm{~nm}$ on fungal biofilms developed on nail fragments, showing good results (22).

While light-based technologies as PDT and IPL are a promising modality for the treatment of Onychomycosis and an alternative for patients who suffer from chronic illness (hepatitis, diabetes, immunosuppression), the literature on PDT and IPL for this treatment is mainly comprised of small studies and case reports. We proposed a short- and medium-term controlled clinical trial to assess and compare the efficacy of a PDT mediated by Methylene Blue (MB) and an IPL system in Onychomycosis treatment.

\section{METHODS}

\subsection{Participants}

A randomized controlled trial (RCT) was designed to achieve the proposed objectives undertaken according to CONSORT guidelines (Supplementary Fig.1). Forty-six patients with all types of toenail Onychomycosis (Table 1) (severe and mild-to-moderate -Table 2-, with a mean chronicity of 3-5 years Table 3- ) in the first toe, with mycological diagnosis of Onychomycosis (PAS (+) Culture (+)), were randomly allocated into two different treatment groups by means of a computer-generated random list: group 1: PDT or group 2: IPL. Patients fulfilling the following criteria were enrolled: prior treatment with unsuccessful oral and / or topical antifungal agents, presence of comorbidity and / or poly-medication that could generate risk when combined with systemic antifungal agents, negative to undergo antifungal treatment. Exclusion criteria were the following: under 18 years of age, contraindications for the use of $\mathrm{MB}$, any disease that can cause nail abnormalities and interfere with evaluation (as for example tinea pedis, psoriasis or lichen planus), pregnancy, lactation, use of oral or systemic antifungals within 4 to 24 weeks prior enrollment in the trial.

After drop-out and exclusion of incomplete recorded patients, the final study population consisted of 40 subjects (13 men and 27 women), and finally each group were constituted by 20 patients. Group 1 PDT: 4 men and 16 women, mean age: $64.4 \pm 17.3$; Group 2 IPL: 9 men and 11 women, mean age: $64.1 \pm 11.4$ (see Supplementary Fig. 1). 
This RCT was granted Ethical Committee approval by the Hospital Clínico San Carlos in Madrid, Spain (Internal code: 17/501-E) in accordance with the ethical principles declared by the World Medical Association of Helsinki (2013) (23). All patients signed an informed consent form before their inclusion in the study.

\subsection{Treatment}

\subsubsection{Pre-treatment}

Nail plates were pre-sliced by a podiatrist and then softened with urea $40 \%$ to increase the penetration of MB and thus the laser radiation. Upon application of urea $40 \%$, the nail was covered with occlusive dressing for 12-24 h. Care was taken not to excessively macerate the periungual skin: in toenails with hyperkeratosis $>2 \mathrm{~mm}$, the softening treatment was applied daily for 7 days before treatments. In toenails with hyperkeratosis $<2 \mathrm{~mm}, 3$ days of softening treatment with urea, was enough.

\subsubsection{Treatment}

The sequence of treatment was: The urea residue from the nail plate and the surrounding skin was cleaned using 70\% alcohol. Nail remnants and hyperkeratosis were mechanically removed from treatment areas with a scalpel or abrasive tool. The nail plate and surrounding skin was again cleaned with $70 \%$ alcohol.

During laser / light application, eye protection of the patient and dermatologist was assured by wearing safety glasses. PDT or IPL procedure took place every 1-2 weeks for up to 8 sessions (so treatment duration was $\sim 16$ weeks). Patients were instructed to disinfect the footwear with formalin tablets.

\subsubsection{Photodynamic therapy}

MB (at a concentration of $2 \%$ in aqueous solution) was applied to the nail and perimeter area, leaving the solution in contact with the nail for $3 \mathrm{~min}$. Next, the MB treated area was irradiated with a low-power diode laser $(\lambda=670 \mathrm{~nm}, 200 \mathrm{~mW}$ - the power was checked prior to each treatment by the use of a Power Meter Laser UNO by People-EO), scanning the affected area of the nail during an exposure time of 5 min. Table 4 provides a brief description of PDT protocol followed in the present study.

\subsubsection{Intense pulsed light}


After assessment of the size and thickness of the infected nail, and ultrasound gel was applied on the plate of the nail under treatment. Then, the area affected was irradiated with the IPL system (Dye-VL-F module tip, Alma Lasers; 10 pulses at $\lambda=500-600 \mathrm{~nm}$, fluence of $10 \mathrm{~J} / \mathrm{cm}^{2}$, pulse duration of $3 \mathrm{~ms}$, irradiated spot area of $1 \mathrm{~cm}^{2}$, pulse repetition rate $1 \mathrm{~Hz}$ ) (Table 4). The module tip was in contact and perpendicular to the nail plate when the treatment was applied. Previously, a clean, white tape was taped on the affected nail's surrounding skin in order to protect the skin from exposure to the strong light emitted by the system during treatment. A pyroelectric detector Gentec-DE 500+ (in combination with Gentec-EO SOLO consola) allowed to perform energy measurements of the light radiation, checked prior to each treatment.

\subsection{Onychomycosis Severity Index (OSI)}

The Onychomycosis Severity Index (OSI) was determined by multiplying the percentage of the affected area (score of 0-5) by the score of the proximity to the matrix (1-5), adding 10 points for longitudinal striae, patches (Dermatophytoma) or subungual hyperkeratosis greater than $2 \mathrm{~mm}$. The result allows classifying the condition as: mild (a score 1-5), moderate (6-15) and severe (16-35).

The microbiological and clinical outcomes were assessed at the end of treatment.

\subsection{Statistical analysis}

Statistical analysis was performed with a SPSS software package for Windows (version 17.0; SPSS, Chicago, III). OSI variable was analyzed by two-factor repeated measures ANOVA (intrasubject factor (time) and an intersubject factor (procedure)). Post-hoc correction to intergroup comparison were tested by Student's t-test and Mann-Whitney test. For intragroup comparison, ANOVA with multiple rank test were used to identify differences between successive follow-ups. A $p$ value of $<0.05$ was considered as statistically significant.

\section{RESULTS}

Of the 46 patients initially enrolled in the present RCT, 40 completed the study. Six patients were not included in the analysis as they did not concluded the protocol (see Supplementary Fig. 1). The 6 dropouts did not clearly unbalanced the initial established groups, as noticed regarding age, severity (Table 2) and chronicity (Table 3), so any restructuration was not needed. Results of the present work showed an improvement in the clinical and mycological status of the treated nails during the treatment. In 
addition, a greater improvement was observed at 12 weeks after the stop of the PDT and IPL sessions ( 28-30 weeks from baseline). Complications or side effects were not observed during or after both procedures. MB showed a good penetration into the nail plate in all patients. In the case of treatment with IPL system, patients felt pain after the application of the last 6-7 pulses per session as consequence of the high energy accumulated in the affected area. In addition, in the IPL group, the apparition of hematomas under nail plate was reported in some cases. These hematomas were reabsorbed approximately after four weeks from their apparition.

Figure 1 and 2 show the evolution of the clinical responses in three toenails from three different patients after PDT and IPL treatment, respectively. The clinical improvement increased progressively from the start of the treatment to 12 weeks post-treatment.

Table 2 and Figure 3 show the evolution of the severity of the disease and OSI scores respectively, during the period analyzed. The treatment was effective in reducing the OSI scores from the start of the PDT procedure: $13.1 \pm 8.6$ (baseline); $6.2 \pm 6.4$ (at 16 weeks follow-up) to $4.1 \pm 4.7$ (at 28 weeks follow-up) (Fig. 3). In the case of IPL treatment, OSI scores were effectively reduced from $17.3 \pm 7.9$ (baseline); $4.1 \pm 2.1$ (at 16 weeks follow-up) to $2.7 \pm 2.0$ (at 28 week follow-up) (Fig.5). OSI values showed the same trend into each group, with a statistically significant decrease $(\mathrm{p}<0.05)$ at 16 and 28 weeks compared to baseline (Fig. 3). However, there was a statistical significant difference as for the evolution over time of OSI scores in both groups: a greater decrease in OSI values during the period ranged between 0-16 weeks in the IPL group $(\mathrm{p}<0.05)$. In both cases, OSI values changed from severe-moderate onychomycosis before the study to slightly mild onychomycosis or clear nail at the end of the study (Table 2).

Table 5 shows the evolution of the mycological response during the period analyzed. Mycological infection widely diminished during the treatment. In 14 patients some remnant dermatophytes were detected by PAS staining after 8 sessions of PDT/IPL (PAS (+) Culture (-): 7 in Group 1 and other 7 in Group 2), however, the analysis after 12 weeks post-treatment confirmed total fungus disappearance in 2 of these patients in the Group 1 and 3 of these patients in the Group 2. Complete cure is the addition of clinical $(0 \%$ in the plate of affection of the nail) and mycological (negative culture and negative histology) response. Results of the present RCT showed that complete cure took place in the $50 \%$ of patients after 8 PDT sessions and in the $70 \%$ after 12 weeks post-treatment, while in the IPL group, 
complete cure took place in the $60 \%$ of patients after 8 PDT sessions and in the $80 \%$ after 12 weeks posttreatment.

\section{DISCUSSION}

Recently, novel therapies based in laser and light have been explored as a possible alternative treatment for Onychomycosis, but, to date, these studies are limited and the mechanism of action remains unclear. Selective destruction of fungal structures seems to be the mechanism of action of these novel therapies (10). The majority of lasers explored for the Onychomycosis treatment are absorbed by water and thus lead to bulk heating (1,064 nm Nd:YAG laser) (24). However, Q-switched 1,064nm Nd:YAG laser, with pulse duration in the nanosecond range seems to be correlated with action on selective targets as melanin, hyphae, micronidia (9). In an in vitro study examining the antibacterial effects of the Q-switched frequency doubled Nd:YAG 532nm laser (presumably absorbed by xanthomegnin), the rate of growth of T. rubrum was slowed (11). IPL system here used as for $\lambda=500-600 \mathrm{~nm}$ and pulse duration $3 \mathrm{~ms}$ was also presumably absorbed by xanthomegnin, although it can be possible that xanthomegnin is not the only target but other untapped target chromophores would be more effective. The IPL system here used with the capability of delivering destructive high energy pulses to specific targets with minimized surrounding tissue damage would seem to be well suited for the task of eradicating organisms on the skin surface.

During the last decade an increase can be seen in research and applications opportunities of antimicrobial PDT (25). This development could be related by the worldwide increasing problem of microbial resistance to commonly used antibiotics. Fungal resistance to currently used antifungal drugs has also been described particularly in case of the dermatophyte $T$ rubrum (26). This therapeutic failure (oral antifungal agents) besides an increased incidence of dermatophyte fungal infections due to a growth of the number of patients with a compromised immune system or the patients suffering diabetes mellitus has contributed to an ever negative image associated with fungal infections $(27,28)$. This negative image associated with Onychomycosis justifies current efforts to develop new treatment modalities like those based on PDT principles. PDT has been shown to be effective in photoinactivation of multiple species of fungi including the most common cause of Onychomycosis T. rubrum (29-31). Development of antimicrobial resistance after PDT treatment appears to be unlikely since, in microbial cells, singlet 
oxygen and other free radicals -all of them originated from this type of therapy- affect a range of cellular targets, something that does not happen in conventional antimicrobial therapy (32).

The results of the present study show a clear improvement in all the patients after both treatments modalities. Those patients who presented a greater affection of the nail had a slower response but also positive. After 12 weeks post-treatment in the PDT group, the percentage of complete, mycological and clinical cure was $70 \%, 70 \%$ and $80 \%$, respectively. In the IPL group, 12 weeks post-treatment, the percentage of complete, mycological and clinical cure was $80 \%, 80 \%$ and $90 \%$, respectively. Most of the $30 \%$ of patients in the PDT group and the $20 \%$ in IPL group, who did not obtain complete cure at 12 weeks post-treatment, showed a good prognosis of the onychomycosis that could be eradicated with adjunctive topical therapy.

Most of the published studies on PDT in Onychomycosis treatment are based on the use of ALA (5aminolevulinic acid) or MAL (methyl aminolevulinate) as photosensitizer, as for example the works of Harris and Pierpoint 2012; Kamp et al. 2005; Gilaberte et al., 2017 (33-35). However, PDT mediated by MB has been proven in a head-to-head trial to be better than the standard of care (36). MB is well known to be photodynamically active, in contrast with ALA or MAL which act as pro-drugs when taken up by the target inducing the accumulation of endogenous PS and, consequently MB allows a quite immediate PDT (very short incubation time of 3 min compared to 3-5h of ALA or $3 \mathrm{~h}$ for MAL) without the needed of using occlusive dressing. In addition, advantages of MB include its low cost, routinely available in the hospital, penetrates well in the nail pretreated with urea and has fewer side effects that ALA or MAL such as less irritation of the skin surrounding the nail or less pain perception. MB is a widely known histological dye that has been in use for many years (37). The characteristic color of MB is caused by the strong absorption band in the $550-700 \mathrm{~nm}$ region with maximum molar absorptivity of $85,000 \mathrm{M}^{-1} \mathrm{~cm}^{-1}$ at $664 \mathrm{~nm}(38,39)$. In the present study a $2 \%$ solution of $\mathrm{MB}$ in water was employed, following the successful pattern described by Souza et al. in their three published woks $(29,30,40)$. Unlike these studies, in the Goup 1 of the present work, MB was photoactivated with laser radiation at 670nm (very near to its peak of maximum absorption $-668 \mathrm{~nm}-$ ), instead of the LED lamp at $630 \mathrm{~nm}$ used by them. The positive effect obtained in this Group may be due by the sum of effects originated by the photodynamic treatment per se and by the biomodulator effect of the red wavelength $(\lambda=670 \mathrm{~nm})$ from the low power diode laser employed. The literature suggests that red light is indeed efficacious at accelerating the electron transport in the mitochondria leading to higher ATP production since red light is 
absorbed by the recognized photoacceptor, cytochrome C oxidase $(41,42)$. This extra of ATP stimulate ROS generation increasing the antifungal activity of polymorphonuclear cells PMN, reported by Cerdeira et al. (43). Immune cells play a crucial role in virtually all types of infections, and need to be effective against a wide range of bacteria, viruses and fungi. Cerdeira et al. show how specific and safe low-level light parameters can boost the innate immune response towards an opportunistic fungus, what may possibly help treating clinical fungal infections in the near future.

Before carrying out either treatment (PDT or IPL), a pretreatment of affected nail with $40 \%$ urea and mechanical removal of nail residue and of hyperkeratotic areas is an important procedure in order to favor both the penetration of the photosensitizer (35) and to help radiation (low-level laser or, intense pulsed light) penetrate under the nail plate to reach the fungal colonies of the nail bed and nail matrix, thereby improving the clinical response to PDT and IPL $(14,31,44)$. The severity index of Onychomycosis graduates the extension and allows predicting the evolution of the affection, thus allowing establishing the degree of affection according to the clinical findings to clinically determine the severity of the Onychomycosis, to guide the therapeutic choice and to predict the result of treatment (2). The use of indexes as OSI makes comparison easier between the results of the present work with those obtained in the other published works. Nowadays, discussing the results obtained is a complicated task because most studies on PDT/IPL and Onychomycosis show different protocols. In the literature there is discrepancy in the duration of treatment, number of sessions applied. Controversy also exists on whether the unit of analysis is the patient (and therefore all of their affected nails) or a single nail per patient. Other studies do not separate the results based on whether the treatment has been performed on the toenails or fingernails and this is important because their average growth rate is different (45). Finally, there are studies that evaluate the mycological cure, others the clinical cure and others the complete cure (46). In order to reduce the number of variables to take into account, we have included patients with a single infected nail of first toe. We have recorded the OSI values as the treatment progressed. Number of patient with clear nail or very mild affectation was correlated with clinical cure. Number of patients that showed PAS (-) and culture (-) was correlated with mycological cure. With the intersection of these values we obtained the percentage of complete cure.

There are still remaining questions about PDT and IPL in Onychomycosis, such as appropriate number of sessions of PDT or IPL to obtain the complete cure, appropriate exhibition time or energy density, rate of recurrence or estimation of long-term maintenance therapy in order to avoid recurrence (47). Another 
important issue to consider if we want to introduce a new treatment in the clinical practice is the cost of the treatment and its duration. While oral therapy comes to last about 6 months with a cost of 20 euros / month (48), MB-mediated PDT therapy or IPL procedure are expected to last 4-8 months, and the cost of the MB solution and of the maintenance of the low level power diode laser or IPL system are minimal. This study can support PDT or IPL as part of the routine dermatological practice in the treatment of Onychomycosis, either as monotherapy or complementing conventional therapies, and the cost of its use is lower than the benefits that it provides.

\section{CONCLUSIONS}

This RCT study confirms that MB/PDT or IPL systems are safe, effective and well tolerated and promote a positive outcome in the treatment of Onychomycosis caused by a wide spectrum of different kind of agents. Further studies are needed to define optimal parameters of actuation (light source (laser/LED), fluence/radiance, light exposure time, number and frequency of treatments required, optimal photosensitizer and its optimal concentration..., to adjust the therapeutic outcome in accordance with the Onychomychosis involvement degree. 


\section{REFERENCES}

[1] Grossberg R. Chapter 4: Fungal diseases of the skin. Bope ET and Kellerman RD (Eds): Conn's Current Therapy. $1^{\mathrm{a}}$ ed. Saunders, Elsevier 2011, USA

[2] Carney C, Tosti A, Daniel R, et al. A new classification system for grading the severity of Onychomycosis: Onychomycosis Severity Index. Arch Dermatol 2011; 147(11):1277-1282.

[3] Gupta AK, Shear NH. Onychomycosis: going for the cure. Can Fam Physician 1997; 43:299-305.

[4] Scher RK, Tavakkol A, Sigurgeirsson B, et al. Onychomycosis: Diagnosis and definition of cure. $J$ Am Acad Dermatol 2007; 56(6):939-944.

[5] Elewski BE, Hay RJ. Update on the management of onychomycosis: highlights of the third annual international summit on cutaneous antifungal therapy. Clin Infect Dis 1996; 23(2): 305-13.

[6] Cohen PR, Scher RK. Topical and surgical treatment of onychomycosis, J Am Acad Dermatol 1994; 31: S74- S77.

[7] Gupta AK, Daigle D, Paquet M. Therapies for onychomycosis a systematic review and network metaanalysis of mycological cure. J Am Podiatr Med Assoc 2015; 105(4): 357-366.

[8] Piraccini BM, Sisti A, Tosti A. Long-term follow-up of toenail onychomycosis caused by dermatophytes after successful treatment with systemic antifungal agents. J Am Acad Dermatol 2010; 62(3): 411-14.

[9] Gupta AK, Simpson FC. Medical devices for the treatment of onychomycosis, Dermatol Ther 2012; 25(6): 574-581.

[10] Ortiz AE, Avram MM, Wanner MA. A Review of Lasers and Light for the Treatment of Onychomycosis. Lasers Med Sci 2014; 46:117-124.

[11] Vural E, Winfield HL, Shingleton AW, Horn TD, Shafirstein G. The effects of laser irradiation on Trichophyton rubrum growth. Lasers Med Sci 2008; 23(4):349-353.

[12] Youngchim S, Pornsuwan S, Nosanchuk JD, Dankai W,Vanittanakom N. Melanogenesis in dermatophyte species in vitro and during infection. Microbiol 2011; 157(Pt 8):2348-2356.

[13] Ackroyd R, Kelty C, Brown N, Reed M. The history of photodetection and photodynamic therapy. Photochem Photobiol 2001; 74(5): 656-69.

[14] Lane N. New light on medicine. Sci Am 2003; 288(1): 38-45.

[15] Oleinick NL, Morris RL, Belinchenko I. The role of apoptosis in response to photodynamic therapy: what, were, why and how. Photochem Photobiol Sci 2002; 1(1): 1-21.

[16] Kessel D, Vicente MG, Reiners JJ. Initiation of apoptosis and autophagy by photodynamic therapy. Autophagy 2006; 2(4): 289-290.

[17] Watanabe D, Kawamura C, Masuda Y, Akita Y, Tamada Y, Matsumoto Y. Successful treatment of toenail onychomycosis will photodynamic therapy. Arch Dermatol 2008; 144(1): 19-21.

[18] Silva AP, Kurachi C, Bagnato VS, Inada NM. Fast elimination of onychomycosis by hematoporphyrin derivative-photodynamic therapy. Photodiagn Photodyn Ther 2013; 10 (3): 328-330.

[19] Dai T, Fuchs BB, Coleman JJ, et al. Concepts and principles of photodynamic therapy as an alternative antifungal discovery platform. Front Microbiol 2012; 3:120.

[20] Ash C, Town G, Whittall R, Tooze L, Phillips J. Lasers and intense pulsed light (IPL) association with cancerous lesions. Lasers Med Sci 2017; 32:1927-1933. 
[21] González-Rodríguez AJ, Lorente-Gual R. Current indications and new applications of intense pulsed light. Actas Dermosifiliogr 2015; 106(5):350-364.

[22] Vila TV, Rozental S, de Sá Guimarães CM. A new model of in vitro fungal biofilms formed on human nail fragments allows reliable testing of laser and light therapies against onychomycosis. Lasers Med Sci 2015; 30(3):1031-1039.

[23] World Medical Association Declaration of Helsinki: ethical principles for medical research involving human subjects. World Medical Association. JAMA. 2013; 310(20): 2191-2194.

[24] Kozarev J, Vizintin Z. Novel laser therapy in treatment of onychomycosis. J Laser Health Acad 2010; 1:1-8.

[25] Hamblin MR, Hasan T. Photodynamic therapy: A new antimicrobial approach to infectious disease? Photochem Photobiol Sci 2004; 3(5): 436-450.

[26] Peres NT, Sanches PR, Falcão JP, et al. Transcriptional profiling reveals the expression of novel genes in response to various stimuli in the human dermatophyte Trichophyton rubrum. BMC Microbiol $2010 ; \mathbf{1 0}, 39$.

[27] Ramos-E-Silva M, Lima CM, Schechtman RC, Trope BM, Carneiro S. Superficial mycoses in immunodepressed patients (AIDS). Clin Dermatol 2010; 28 (2): 217-225.

[28] Gupta AK, Humke S. The prevalence and management of onychomycosis in diabetic patients. Eur J Dermatol 2000; 10(5): 379-384.

[29] Souza LWF, Souza SVT, Botelho ACC. Endonyx toenail onychomycosis caused by Trichophyton rubrum: treatment with photodynamic therapy based on methylene blue. An Bras Dermatol 2013; 88(6):1019-1021.

[30] Souza LWF, Souza SVT, Botelho ACC. Distal and lateral toenail onychomycosis caused by Trichophyton rubrum: treatment with photodynamic therapy based on methylene blue due. An Bras Dermatol 2014; 89(1): 184-186.

[31] Piraccini BM, Rech G, Tosti A. Photodynamic therapy of onychomycosis caused by Trichophyton rubrum. J Am Acar Dermatol 2008; 59 (5 Suppl): S75-S76.

[32] van Straten D, Mashayekhi V, de Bruijn HS, Oliveira S, Robinson DJ. Oncologic photodynamic therapy: basic principles, current clinical status and future directions, Cancers 2017; 9:19.

[33] Harris F, Pierpoint L. Photodynamic therapy based on 5-aminolevulinic acid and its use as an antimicrobial agent. Med Res Rev 2012; 32 (6):1292-1327.

[34] Kamp H, Tietz HJ, Lutz M, et al. Antifungal effect of 5-aminolevulinic acid PDT in Trichophyton rubrum. Mycoses 2005; 48(2):101-107.

[35] Gilaberte Y, Robres MP, Frías MP, García-Doval I, Rezusta A, Aspiroz C. Methyl aminolevulinate photodynamic therapy for onychomycosis: a multicentre, randomized, controlled clinical trial. J Eur Acad Dermatol Venereol 2017; 31(2):342-354.

[36] Simmons BJ, Griffith RD, Falto-Aizpurua LA, Nouri K. An update on photodynamic therapies in the treatment of onychomycosis. J Eur Acad Dermatol Venereol 2015; 29(7): 1275-1279.

[37] Tuite EM, Kelly JM. Photochemical interactions of methylene blue and analogues with DNA and other biological substrates. J Photochem Photobiol B 1993; 21(2-3):103-124.

[38] Junqueira HC, Severino D, Dias LG, Gugliotti M, Baptista MS. Modulation of the methylene blue photochemical properties based on the absorption at aqueous micelle interfaces. Phys Chem Chem Phys 2002; 4: 2320-2328. 
[39] Tardivo JP, Del Giglio A, Oliveira CS, et al. Methylene blue in photodynamic therapy. From basic mechanisms to clinical applications. Photodiagn Photodyn Ther 2005; 2:175-191

[40] Souza LWF, Souza SVT, Botelho ACC. Randomized controlled trial comparing photodynamic therapy based on methylene blue dye and fluconazole for toenail onychomycosis. Dermatol Ther 2014; 27:43-47.

[41] Pastore D, Greco M, Passarella S. Specific helium-neon laser sensitivity of the purified cytochrome c oxidase. Int J Radiat Biol 2000; 76: 863-870.

[42] Eells JT, Henry MM, Summerfelt P, et al. Therapeutic photobiomodulation for methanol-induced retinal toxicity. Proc Natl Acad Sci USA 2003; 100 (6), 3439-3444.

[43] Cerdeira CD, Lima Brigagão MR, Carli ML, et al. H. Low-level laser therapy stimulates the oxidative burst in human neutrophils and increases their fungicidal capacity. $J$ Biophot 2016; 9(11-12): 1180-1188.

[44] Kalokasidis K, Onder M, Trakatelli MG, Richert B, Fritz K. The effect of Q-switched Nd:YAG $1064 \mathrm{~nm} / 532 \mathrm{~nm}$ laser in the treatment of onychomycosis in vivo. Dermatol Res Pract 2013; Article ID 379725,10 pages.

[45] US Food and Drug Administration. 2015. Medical Devices and Clinical Trial Design for the treatment or improvement in the appearance of fungally-infected nails- Draft guidance for industry and FDA staff (Internet). URL http://www.fda.gov/downloads/MedicalDevices/DeviceRegulationandGuidance/GuidanceDocuments/UC M431312.pdf

[46] Gupta AK, Versteeg SG. A critical review of improvement rates for laser therapy used to treat toenail onychomycosis. J Eur Acad Dermatol Venereol 2017; 31(7): 1111-1118.

[47] Amorin JC, Soares BM, Alves OA, et al. Phototoxic action of light emitting diode in the "in vitro" viability of Trichophyton rubrum. An Bras Dermatol 2012; 87(2):250-5.

[48] Mikailov A, Cohen J, Joyce C, Mostaghimi A. Cost-effectiveness of confirmatory testing before treatment of onychomycosis. JAMA Dermatol 2016; 152: 276-281. 


\section{Figure Legends}

Figure 1- Three toenail onychomycosis treated with photodynamic therapy mediated by $2 \%$ MB. (A) Before treatment; (B) after treatment with 8 PDT-sessions; (C) at 12 weeks after PDT treatment.

Figure 2- Three toenail onychomycosis treated with IPL system. (A) Before treatment; (B) after treatment with 8 IPL-sessions; (C) at 12 weeks after IPL treatment.

Figure 3.- Evolution of OSI scores during the study. (*) Significance of intragroup differences compared to baseline; $\left({ }^{\wedge}\right)$ Significance of intergroup differences with respect the evolution over time (0-16 week). 
Table 1.- Causative agent of the disease among the sample population.

\begin{tabular}{l|c}
\hline Kind of microorganism & $\begin{array}{c}\text { Number of patients } \\
\text { affected }\end{array}$ \\
\hline Trichophyton sp & 15 \\
Trichophyton rubrum & 13 \\
Trichophyton mentagrophytes & 6 \\
Candida sp & 2 \\
Candida parapsilosis & 2 \\
Candida famata & 1 \\
Aspergillus fumigatus & 2 \\
Rhodotula & 1 \\
Microsporum & 2 \\
Microsporum+Epidermophyton & 1 \\
Fusarium & 1 \\
\hline
\end{tabular}


PHOTO - manuscript copy

Page 18 of 25

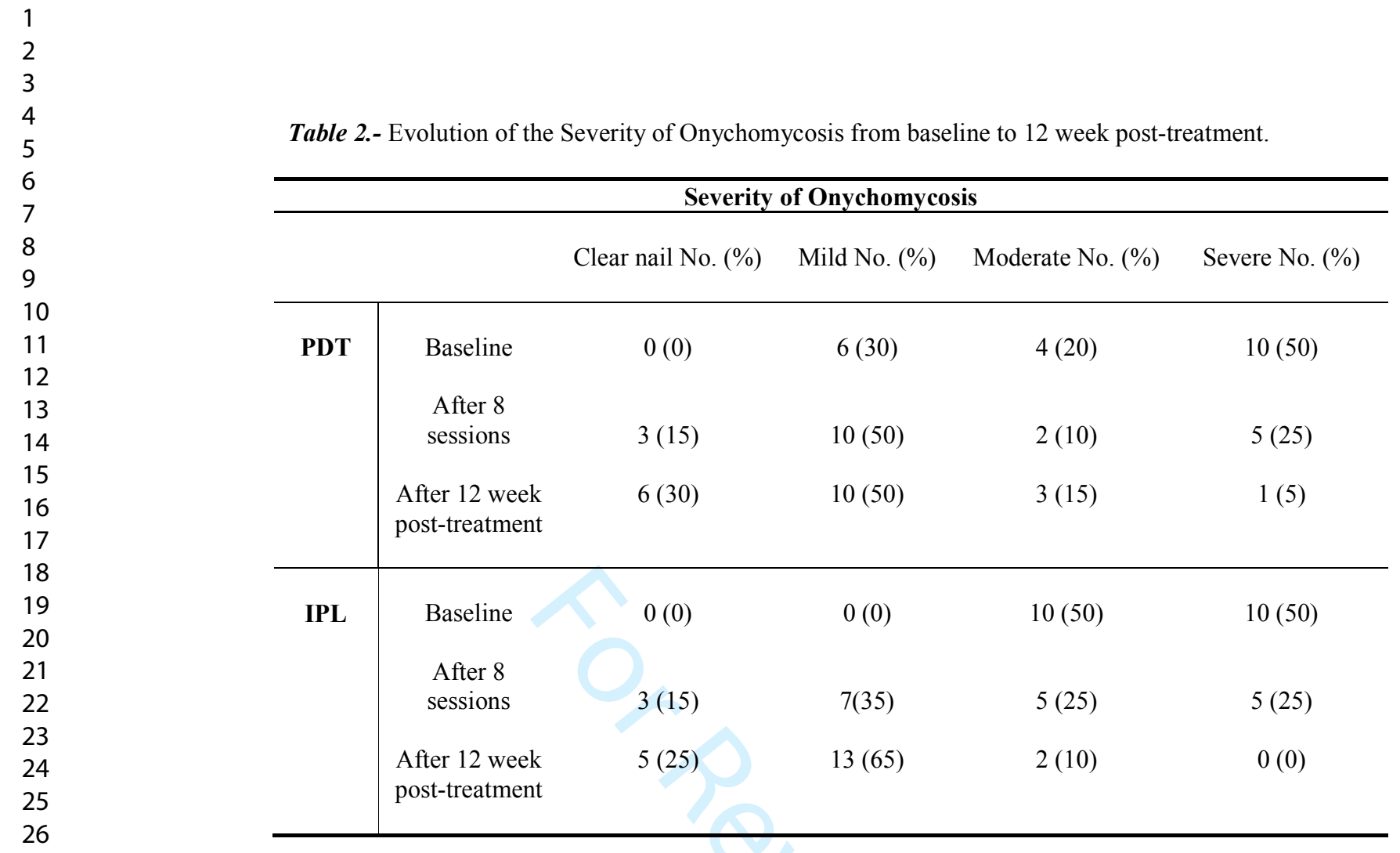

27

28

29

30

31

32

33

34

35

36

37

38

39

40

41

42

43

44

45

46

47

48

49

50

51

52

53

54

55

56

57

58

59

60 
Table 3.- Sample population classified as a function of the chronicity of the disease (No.= number of patients)

\begin{tabular}{lccc}
\hline & \multicolumn{3}{c}{ CHRONICITY } \\
\hline & 2 years No. & 3-5 years No. & 6 years No. \\
\cline { 2 - 4 } PDT & & & \\
\hline Men & - & 2 & 2 \\
Women & 5 & 6 & 5 \\
\hline & & & \\
IPL & & 3 & 3 \\
Men & 3 & 5 & 5 \\
\hline
\end{tabular}


Table 4.- Summary of PDT and IPL protocol and the laser/light parameters used in this study.

\section{PDT}

PS characteristics: solution of MB $2 \% \mathrm{w} / \mathrm{v}$

PS incubation time: $3 \mathrm{~min}$

Photoexcitation wavelength (nm): $670 \mathrm{~nm}$

Irradiation mode: continuous mode

Power output (mW): 200

Irradiance $\left(\mathrm{W} / \mathrm{cm}^{2}\right): 6.37$

Spot diameter $(\varnothing)(\mathrm{cm}): \sim 0.2$

Time of laser radiation exposure: $5 \mathrm{~min}$

Frequency of application: 8 sessions separated by an interval of 2 weeks

IPL

Wavelength (nm): 500-600

Irradiation mode: pulsed mode

Fluence $\left(\mathrm{J} / \mathrm{cm}^{2}\right): 10$

Number of pulses:10

Spot area $\left(\mathrm{cm}^{2}\right): 1$

Pulse repetition rate: $1 \mathrm{~Hz}$

Frequency of application : 8 sessions separated by an interval of 2 weeks

Ps: Photosensitizer; MB: Methylene blue 
Table 5.- Evolution of the mycological response at the end of the 8 session and at 12 week post-treatment (No.= number of patients; $(\%)=$ percentage of patients).

\begin{tabular}{|c|c|c|c|c|c|}
\hline & $\begin{array}{c}\text { MYCOLOGICAL } \\
\text { RESPONSE }\end{array}$ & $\begin{array}{c}\text { PAS }(+) \\
\text { Culture }(+) \\
\text { No. }(\%)\end{array}$ & $\begin{array}{c}\text { PAS (+) } \\
\text { Culture (-) } \\
\text { No. }(\%) \\
\end{array}$ & $\begin{array}{c}\text { PAS }(-) \\
\text { Culture }(+) \\
\text { No. }(\%)\end{array}$ & $\begin{array}{c}\text { PAS (-) } \\
\text { Culture (-) } \\
\text { No. }(\%) \\
\end{array}$ \\
\hline \multirow[t]{3}{*}{ PDT } & At baseline & $20(100)$ & $0(0)$ & $0(0)$ & $0(0)$ \\
\hline & At 8 sessions & $1(5)$ & $7(35)$ & $2(10)$ & $10(50)$ \\
\hline & $\begin{array}{l}\text { At } 12 \text { week post- } \\
\text { treatment }\end{array}$ & $0(0)$ & $5(25)$ & $1(5)$ & $14(70)$ \\
\hline \multirow[t]{3}{*}{ IPL } & At baseline & $20(100)$ & $0(0)$ & $0(0)$ & $0(0)$ \\
\hline & At 8 sessions & $0(0)$ & $7(35)$ & $1(5)$ & $12(60)$ \\
\hline & $\begin{array}{l}\text { At } 12 \text { week post- } \\
\text { treatment }\end{array}$ & $0(0)$ & $4(20)$ & $0(0)$ & $16(80)$ \\
\hline
\end{tabular}



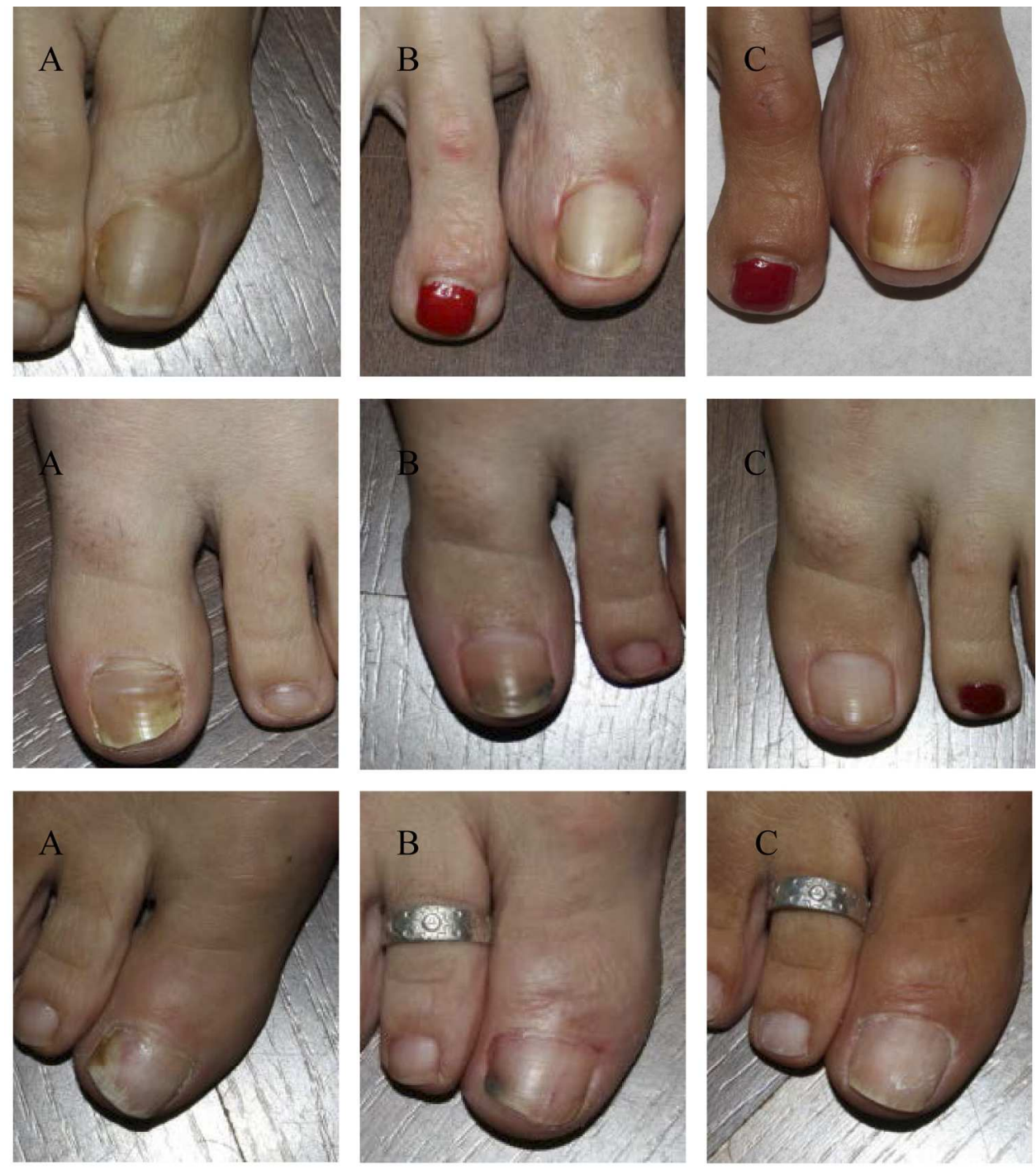

Figure 1

Figure 1 - Three toenail onychomycosis treated with photodynamic therapy mediated by $2 \% \mathrm{MB}$. (A) Before treatment; (B) after treatment with 8 PDT-sessions; (C) at 12 weeks after PDT treatment.

$184 \times 230 \mathrm{~mm}(300 \times 300 \mathrm{DPI})$ 

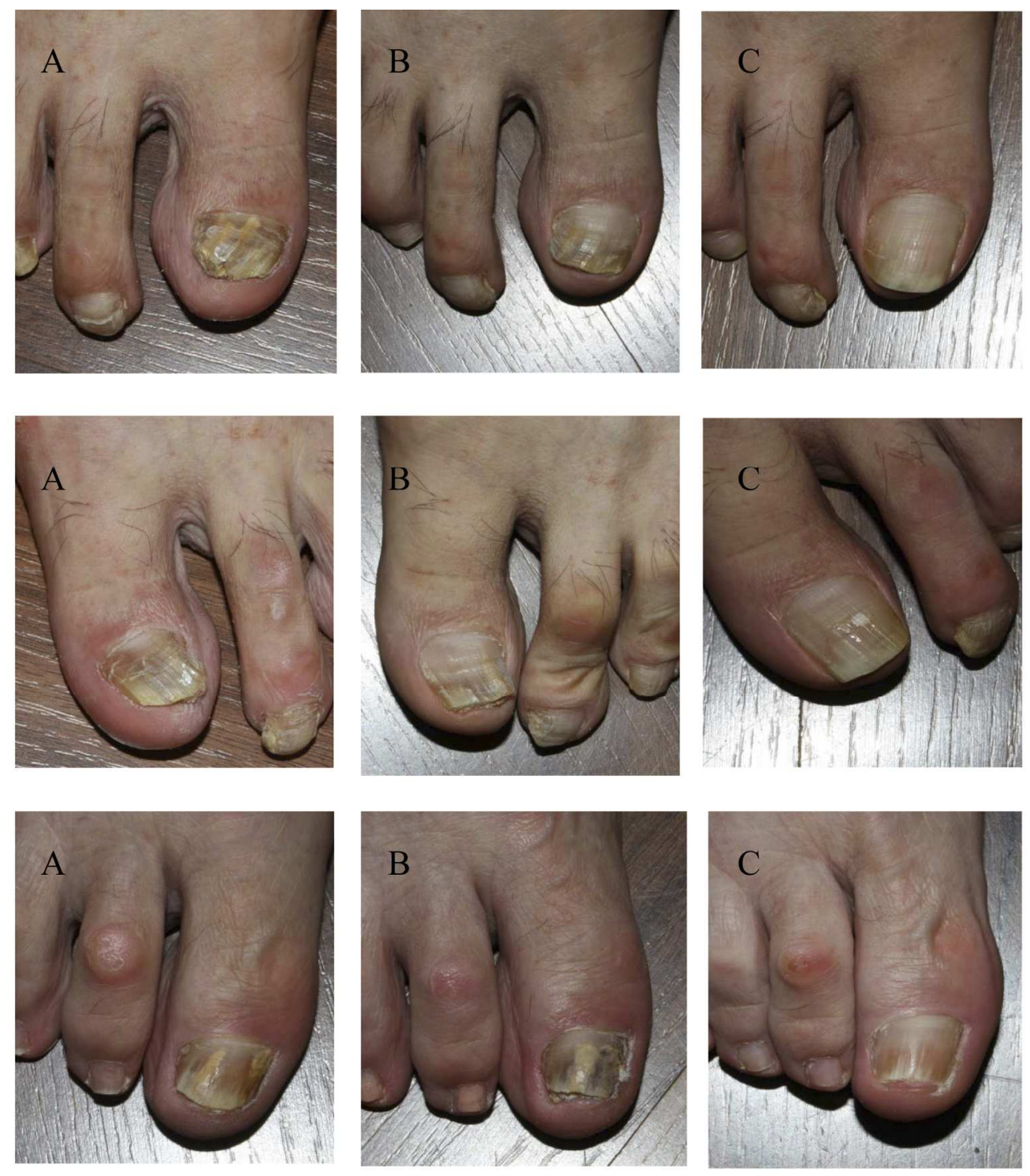

Figure 2

Figure 2- Three toenail onychomycosis treated with IPL system. (A) Before treatment; (B) after treatment with 8 IPL-sessions; (C) at 12 weeks after IPL treatment.

$187 \times 228 \mathrm{~mm}(300 \times 300 \mathrm{DPI})$ 
Figure 3.- Evolution of OSI scores during the study. (*) Significance of intragroup differences compared to baseline; $(\wedge)$ Significance of intergroup differences with respect the evolution over time (0-16 week).

$140 \times 101 \mathrm{~mm}(300 \times 300 \mathrm{DPI})$ 


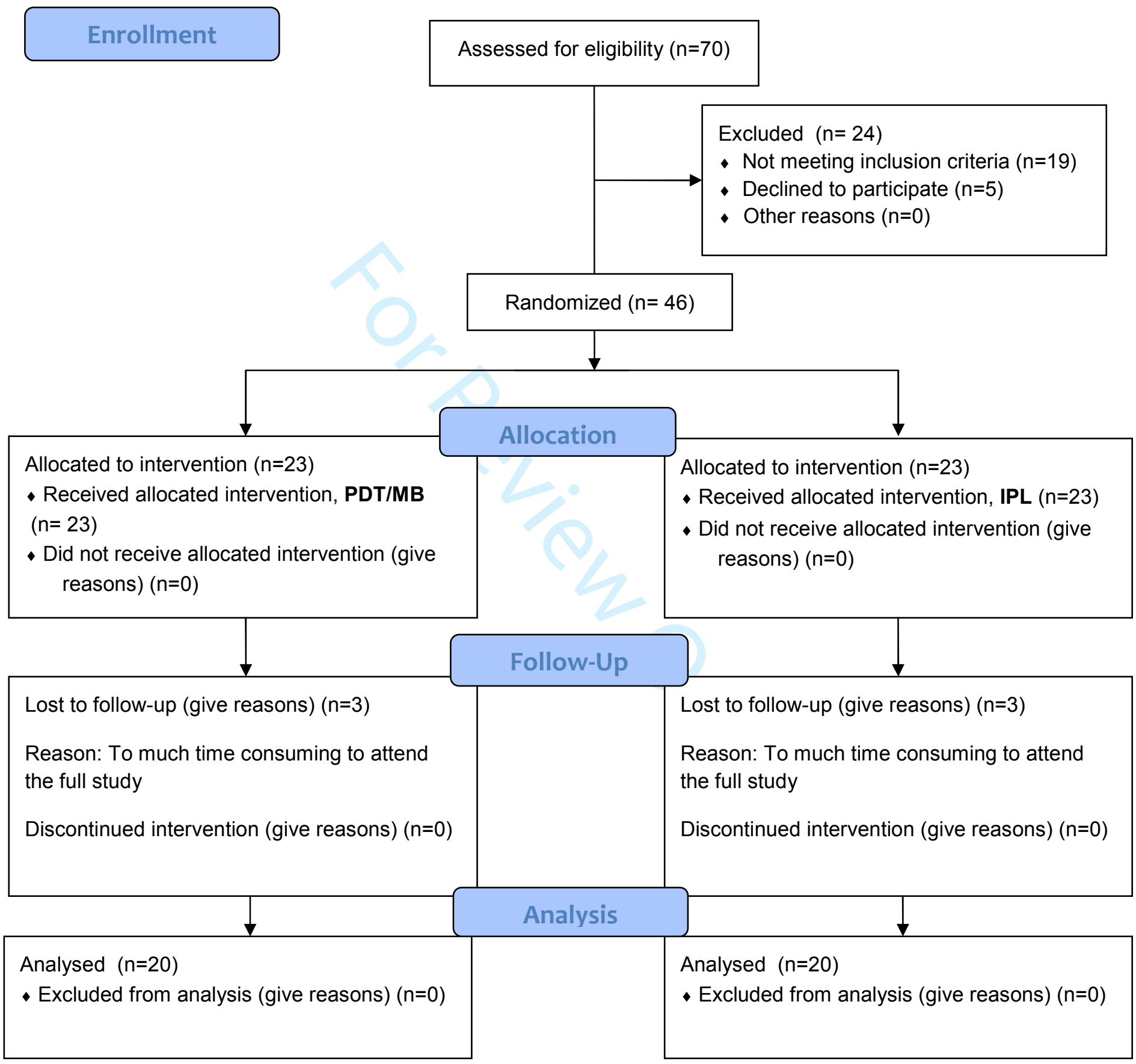

